\title{
Perianal White Sponge Nevus In A Hispanic Female: A Case Study
}

\author{
N Busen, M Tompkins
}

\begin{abstract}
Citation
N Busen, M Tompkins. Perianal White Sponge Nevus In A Hispanic Female: A Case Study. The Internet Journal of Advanced Nursing Practice. 1999 Volume 4 Number 2.
\end{abstract}

DOI: $\underline{10.5580 / 6 c 5}$

\begin{abstract}
This article presents a case history of white sponge nevus (WSN) in the perianal area of a 23-year-old Hispanic female. WSN is a rare, autosomal dominant disorder that affects the noncornified stratified squamous epithelia. Clinically, the lesions are characterized by the presence of white, spongy plaques in the oral cavity. Extraoral lesions are found in other mucosal sites but are relatively uncommon in the absence of oral manifestations. Because WSN is rare, the differential diagnosis of this lesion may be difficult and the diagnosis is best made by a tissue biopsy performed in the context of a detailed patient and family history. The problems associated with differential diagnosis are addressed.
\end{abstract}

\section{INTRODUCTION}

Diagnosing lesions in the genital area by appearance can sometimes prove elusive as illustrated by the case of a 23year-old Hispanic female who presented to an urban college health clinic with a long-standing complaint of a pruritic, white lesion in the anogenital area. Although the patient had numerous diagnoses and treatments over a five year period, the lesion remained unchanged.

\section{CASE REPORT}

\section{SUBJECTIVE DATA}

The patient is a 23-year-old Hispanic female who presents to a college health clinic for the diagnosis and management of a "white, itchy spot" in her genital area. In the past five years, she states that she has been evaluated, diagnosed, and treated for the same condition at several other clinics and private practices. She was previously diagnosed and treated for a yeast infection and anal warts, for this same complaint, with no resolution of symptoms. She has looked up these conditions, for which she was treated, on the internet and does not feel that she was appropriately diagnosed.

\section{PAST MEDICAL HISTORY}

The patient's general health is reportedly good. The patient was successfully treated for chlamydia in 1994 and has had no other sexually transmitted diseases. She started menstruating at age 12 and became sexually active in her late teens. She has been taking oral contraceptive pills (OCPs) intermittently for the past 5 years when sexually active. She reports a monogamous relationship at present. She infrequently uses condoms for contraception and would like to start on OCPs again. She does not smoke and denies use of alcohol. She states that her mother (aged 48 years) is currently being treated for an aggressive cervical cancer, a fact that makes her more anxious about her condition. She denies that any other immediate family members have a similar condition or that she has any similar lesions elsewhere on her body.

\section{OBJECTIVE DATA}

In a lithotomy position, the lesion is easily visible. The patient has a $3-4 \mathrm{~cm}$ diameter lesion around her anus. The lesion is a white, lichenified, well-defined plague with a ruggated or corrugated surface (See figures1 and 2). A small amount of scar tissue is visible around a partial perimeter of the lesion, which could be a result of past treatment for anogenital warts. There are no other lesions in the genital area on both internal and external exam. There are no other 
lesions that extend into the anal canal or the vaginal vault. The physical and pelvic exam are completely within normal limits. There are no nail or skin abnormalities and no lymphadenopathy. The oral cavity is clean and the teeth are in good condition. No lesions are noted in the nose or throat. The Pap smear obtained shows inflammation for the second time in 15 months.

\section{Figure 1}

Figure 1:

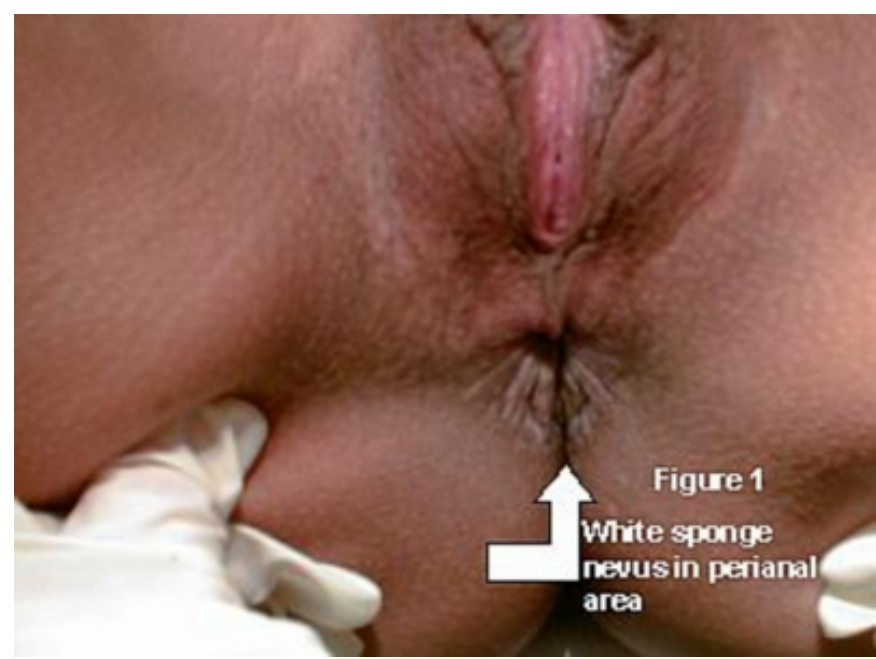

Figure 2

Figure 2

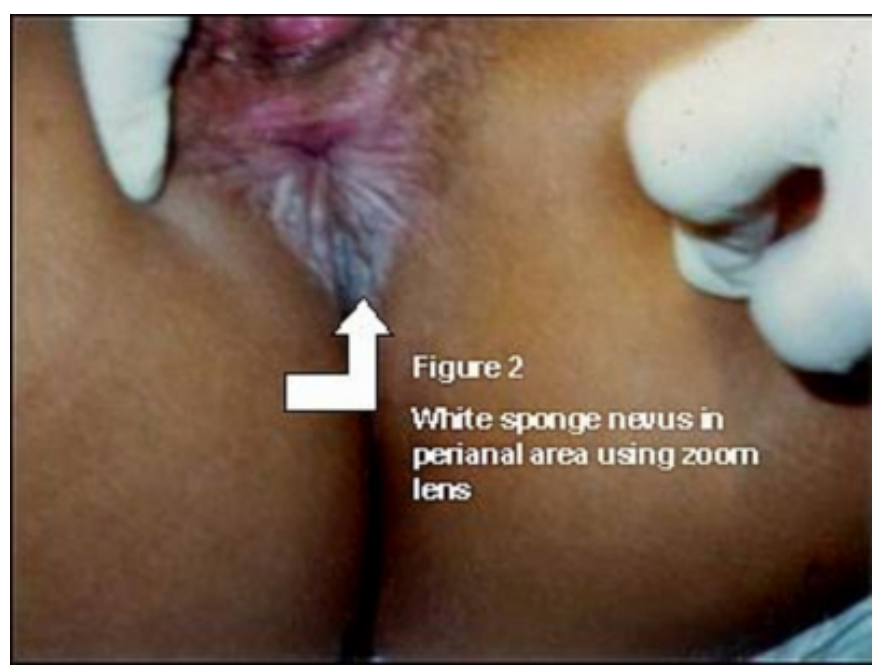

\section{IMPRESSION}

The lesion appeared somewhat dystrophic and was initially thought to be a dystrophic condition, lichen planus, or lichen sclerosis. Given the mother's problems with cervical cancer, and the patient's five-year history of this lesion, the patient was referred.

\section{REFERRAL, CLINICAL DIAGNOSIS AND TREATMENT}

The nurse practitioners who examined this patient felt that she would benefit from an evaluation by a dermatologist who could more definitively diagnose the lesion by biopsy. Although the patient had no insurance and little money, she was evaluated at a local university dermatology service and a $4 \mathrm{~mm}$ punch biopsy of the lesion was obtained. The preliminary diagnosis was white sponge nevus. The pathology report noted parakeratosis, acanthosis and papillomatosis consistent with a diagnosis of white sponge nevus. The patient was informed of the diagnosis and encouraged to keep the area as dry as possible and to use an antipruritic cream such as Aveeno anti-itch cream. She was instructed to monitor the lesion and consult dermatology for any changes. Because the condition is considered benign, no additional treatment was necessary.

\section{EPIDEMIOLOGY}

A search of dermatological and gynecological literature revealed very little about white sponge nevus in the genital area. More information was available on this lesion in the oral cavity and was retrieved from the dermatological and dental literature.

White sponge nevus (WSN) is a relatively rare, autosomal dominant abnormality of the squamous epithelia ${ }_{1,2}$,. If one parent demonstrates this condition, $50 \%$ of future progeny could demonstrate the condition as well. The lesions were first described by Hyde in 19033 and then in 1935, Cannon discussed the clinical and histological aspects and coined the term "white sponge nevus". The condition is congenital, although it may not present (or be discovered) until childhood or adolescence 2 . Males and females are equally affected. Mutations in keratin 4 (K4) and keratin 13 (K13) genes have been shown to be responsible for WSN1. The disease occurs most commonly as mucosal lesions in the oral cavity and is typically found in family members. Penetrance (the percentage of individuals with a mutation that actually demonstrate the phenotypic change) is high $(87 \%)_{5}$ with family involvement reported through five generations 6 . Patients who report no family history of WSN most likely represent a sporadic genetic mutation, and their off-spring would be no more likely to demonstrate the condition than would occur by chance mutation ${ }_{7}$. Less frequently, extraoral sites are involved that include the mucous membranes of the nose, esophagus, rectum, and vulvovaginal area. Rarely, do extraoral lesions occur without oral manisfestations 6 . There is a wide variability in size and location but anogenital 
lesions are relatively rare, with only two cases reported by the current literature review 6,7 .

\section{CLINICAL MANIFESTATIONS}

Clinically, the lesions of WSN present as bilateral, soft, white, spongy plaques on the oral mucosa and extraoral sites and plaques have a folded or corrugated appearance. The plaques are frequently described as having a velvety or rugose surface $2,5,8$. The buccal mucosa is most frequently affected, followed by the labial and gingival mucosa, and the floor of the mouth 5. Plaques may also be present along the lateral borders of the tongue 2 . The plaque is thick and may peel away from the mucosal surface. The white color does not diminish when the tissue is stretched in any mucosal site 2.

The clinical presentation of WSN varies among individuals. Different areas of the oral mucosa and extraoral sites will vary from patient to patient, and the distribution of lesions can change with time in the same patient, although generally lesions remain unchanged 1,9 . Although one case of squamous cell carcinoma was reported arising in a $\mathrm{WSN}_{10}$, the disease is believed to have a benign clinical course1.

The lesions of WSN are generally painless and asymptomatic, although some patients have complained of pruritis (as did our patient), burning (especially after contact by an irritant), and, with oral involvement, dry mouth and roughness of the mouth and tongue $6,7,,_{11}$.

\section{DIAGNOSIS AND MANAGEMENT}

The diagnosis of WSN is made by patient history, family history, and tissue biopsy using a Papanicolaou-stained smear and/or light and electron microscopy1. DNA and RNA extraction may be done with blood from the patient and family members and reverse transcriptase-polymerase chain reaction (RT-PCR) and direct sequencing may be done to detect the exact genotype1,2. Features such as epidermal hyperplasia, acanthosis (hypertrophy of the prickle cell layer), hyperkeratosis, parakeratosis, and intracellular edema (within the cells of the spinous layer of the squamous epithelia) are diagnostic of WSN 2,5,8. There also may be extensive cytoplasmic vacuolization (excessive formation of spaces among the cells), the cell nuclei may be pyknotic (with thickening and shrinkage of the nuclei), and the adjacent tissue normal $5,{ }_{12}$.

\section{ORAL LESIONS}

The differential diagnosis of oral WSN includes consideration of inherited and acquired conditions (See table 1). Pachyonychia congenita, hereditary benign intraepithelial dyskeratosis and Darier disease are inherited mucosal syndromes characterized by white oral plaques but have clinical features that distinguish them from WSN 2,8,12. Pachyonychia congenita and Darier disease are characterized by nail dystrophy and skin involvement; pachyonychia congenita causes hyperkeratosis of the palms and soles, follicular keratosis of the knees and elbows, and hyperhydrosis of the hands and feet 2,8,12. Patients with hereditary benign intraepithelial dyskeratosis develop foamy, gelatin-like plaques on the bulbar conjunctiva that may eventually lead to blindness as a result of vascularization of the cornea 2,12 .

Nonhereditary white lesions of the oral cavity are numerous. The most common lesions that may be confused with WSN are leukoedema, leukoplakia, candida, cheek biting and lichen planus2. Leukoedema has no familial history, a late onset, an intermittent course, is found on the buccal mucosa and occurs primarily in blacks $(90 \%) 2$. There are no extraoral lesions with leukoedema. A clinically diagnostic feature that distinguishes leukoedema from WSN, is that the white lesions disappear when the mucosal tissue is stretched2. Leukoplakia, a milky white, slightly elevated plaque, occurs on the buccal mucosa and is firm in consistency 13.

Leukoplakia generally occurs in adults (40-70 years) and is usually localized to one site. Leukoplakia can be confirmed by biopsy, which shows atypical keratinocytes, abnormal mitosis, cell and nuclear pleomorphism, and enlarged or multiple nucleoli13. Candidiasis involves white "curd-like" lesions, which are easily scrapped off the tongue and bucccal mucosa, and a potassium hydroxide $(\mathrm{KOH})$ preparation demonstrates budding yeast and pseudohyphae14. Cheek biting displays macerated tissue and occurs only in areas of the mouth where the teeth can make contact with the mucosa. Oral lichen planus involves the buccal mucosa bilaterally but presents as an interlacing network of white striae, which differentiates the lesions from WSN; the diagnosis is generally based on clinical presentation13. As in most cases, a good patient history provides the clinician with the best diagnostic clues when distinguishing WSN from other conditions.

\section{GENITAL LESIONS}

The diagnosis of WSN in extraoral sites, such as the anogenital area, requires a differential diagnosis of inherited, 
acquired and/or infectious conditions (See table 2). Like WSN, psoriasis is an inherited skin disorder, but psoriasis is characterized by salmon-pink plaques covered by loose silvery-white scales. Unlike WSN, the scales of psoriasis are easily epilated, leaving miniscule blood droplets (Auspitz phenomena) and psoriasis has a widely variable course with multiple exacerbations and remissions 13 .

Another recurrent genital rash, which is inherited as an autosomal dominant trait, is familial benign pemphigus (Hailey-Hailey disease)15. Onset is usually in adolescence and the disease is characterized by exacerbations and remissions that fluctuate with the seasons. The disease is worse in summer months and can be extremely pruritic. The lesions present as vesicles that rupture easily, leaving crusty erosions resembling white vegetative plaques, particularly in areas such as the axilla and groin 15 .

Benign lesions which could be confused with WSN include lichen planus. Lichen planus is an inflammatory condition of the skin and mucous membranes of unknown etiology characterized by violaceous, shiny, pruritic, flat paules with white lines (Wickham's striae) ${ }_{13}$. Anogenital lichen planus usually presents in adults and is extremely pruritic and sometimes painful16. Patient history, clinical presentation and histology differentiate anogenital lichen planus from WSN.

Acquired conditions such as lichen sclerosis, and infectious conditions such as condylomata acuminata, condylomata lata, and candidia have patient history and clinical features that distinguish these conditions from WSN. Lichen sclerosis is a chronic atrophic, mucocutaneous disorder of unknown etiology that can occur in children, but the mean age of onset is between 40 and 50 years13. Females are most frequently affected, with characteristic lesions of pearly white, angular, indurated papules and plaques primarily in the vulvar and perianal area. Males may have lesions under the surface of the prepuce and glans penis13. Genital symptoms may vary from painful erosion to severe atrophy of anatomical structures, such as vulvar atrophy13,16.

The terms "dystrophy" and "leukoplakia" in the vulvar area have been replaced with "vulvar atypia-intraepithelial neoplasia" 16. Vulvar atypia-intraepithelial neoplasias are staged from mild dysplasia (VIN I) to moderate dysplasia (VIN II) and to severe dysplasia (VIN III) depending on which level of the squamous epithelium is involved16. Carcinoma in situ involves the full thickness of the epithelium. The tissue in the vulvar area may appear white or reddish and patients may complain of pruritis. Vulvar atypias can be definitively diagnosed by biopsy and differentiated miscrospically from WSN by the size and variation of the nuclei16. Condylomata acuminata and condylomata lata are infectious conditions caused by human papilloma virus (HPV) and T. pallidum (a spirochete) respectively $13,{ }_{14}$. Condylomata acuminata usually present as small flesh colored papules, or groups of papules, that have a cauliflower-type appearance. Microscopic features of condylomata acuminata may have some similarities with WSN, but WSN can be differentiated by the lack of binucleation and nuclear hyperchromasia typical of condylomata acuminata7.

Condylomata lata is characterized by large clusters of brownish-red or pink, moist papules and is easily diagnosed based on history, presentation and a serologic test for syphilis13,14. Mucous patches on the oral or genital mucoa may also characterize secondary syphilis. These lesions are flat macules and papules covered by a white-gray membrane14,16.

Mucocutaneous candidiasis in the anogenital area is caused by candida albicans which shows branching peudohyphae and budding yeast on a $\mathrm{KOH}$ preparation which easily distinguishes this condition from WSN13. The lesions are usually bright red, confluent plaques with red satellite papules and a fine white collar13. Candida can occur at any age and may be associated with a thick, creamy white, curdlike discharge in the mouth and vaginal areas.

\section{TREATMENT}

The plaques of WSN are considered benign and no treatment is usually indicated because the lesions are generally asymptomatic6,11,,$_{17}$. In some individuals, where the lesions interfere with normal function and/or cause sufficient discomfort, treatment may be instituted. Treatment of oral WSN includes the use of topical $0.05 \%$ tretinoin and/or topical antibiotics, such as $0.25 \%$ tetracycline or penicillin, although results are variable $5,8,11$. In one documented case, surgical removal of a small discrete WSN from the labia minora was successful6. Comfort measures, such as mouth rinses, are helpful with associated symptoms of WSN in the oral cavity and anti-pruritic creams may be applied to anogenital lesions. Cotton underwear and loose fitting clothing help to decrease warmth and moisture in the genital area. Keeping the area clean and dry also decreases irritation that may contribute to pruritis and burning. 


\section{SUMMARY}

Although WSN more frequently involves the oral cavity, and rarely affects extraoral sites, the diagnosis of WSN should be considered in cases where more typical genital lesions are not suspected. A detailed patient and family history will provide good diagnostic clues for the practitioner, especially if other treatments fail to improve the clinical condition.

Referral for more definitive testing is generally an option and certainly proved to be helpful in the diagnosis of this case.

\section{ACKNOWLEDGMENT}

The authors wish to thank Gloria Mendoza, The University of Texas-Houston School of Nursing, for typing this manuscript. Great appreciation is expressed to our patient, who graciously shared her history, and allowed us to photograph her lesion and to use the pictures for educational purposes. We also wish to acknowledge Dr. Adelaide Herbert, The University of Texas-Houston Health Science Center, Department of Dermatology, who diagnosed the lesion of WSN in this patient.

\section{TABLE 1. CAUSES OF ORAL LEUKOPLASIA}

\section{$2,8,12$}

I. Inherited

- White sponge nevus

- Pachyonychia congenita

- Hereditary benign intraepithelial dyskeratosis

- Darier disease

II. Acquired

- Benign focal epithelial hyperplasia

- Leukoedema

- Leukoplakia

- Oral papillomatosis

- Candidiasis

- Lichen planus

- Lichen sclerosis

- Lupus erythematosus
- Chronic irritants (smoking, cheek biting)

TABLE 2. CAUSES OF GENITAL LEUKOPLASIA 9,13,14,

I. Inherited

- White sponge nevus

- Psoriasis

- Familial benign pemphigus (Hailey-Hailey Disease)

II. Acquired

- Lichen planus

- Lichen sclerosis

- Dystrophy

- Leukoplakia

- Psoriasis

III. Infections

- Condylomata acuminata

- Condylomata lata

- Secondary Syphilis (mucous patches)

- Candidiasis

\section{References}

1. Terrinoni A, Candi C, Oddi S, Gobello T, Camaione D, Mazzanti C et al. A glutamine insertion in the $1 \mathrm{~A}$ alpha hellical domain of the keratin 4 gene in familial case of white sponge nevus. J I Dermat 2000;114:388-391.

2. Morris R, Gansler T, Rudisill M, Nevill B. White sponge nevus. Acta Cytol 1988;32:357-361.

3 . Hyde JN. An unusual nevus of the tongue in a five-year old boy. J Cutan Dis 1990;27:256.

4. Cannon AB. White spone nevus of the mucosa. Arch Dermat and Syph 1935;31:365-370.

5. Jorgenson RJ, Levin LS. White sponge nevus. Arch Dermatol 1981;117:73-76.

6. Buckholz F, Schubert C, Lehmann-Willenbrock W. White sponge nevus of the vulva. Int J Gyn Obstet 1985; 23:505-507.

7. Nichols G, Cooper P, Underwood P, Greer K. White sponge nevus. Obstet Gyn 1990;76:545-548.

8. Hernandez-Martin A, Fernandez-Lopez E, de Unamuno P, Armijo M. Diffuse whitening of the oral mucosa in a child. Pediatr Dermatol 1997;44:316-320.

9. Aloi F, Molinero A. White sponge nevus with epidermolytic changes. Dermatol 1988; 177:323-326. 
10. Downham TF, Plezia RA, Oral squamous cell carcinoma with a white-sponge nevus. J Dermatol Surg Oncol 1978;4:470-472.

11. McDonagh AJ, Gawkrodger DJ, Walker AE. White sponge naevus successfully treated with tropical tetracycline. Clin Exp Dermatol 1990;15:152-153.

12. Witkop CJ, Gorlin RJ. Four hereditary mucosal syndromes. Arch Dermatol 1961;85:762-771.

13. Fitzpatrick T, Johnson RA, Suurmond, D, Wolff, K. Color atlas and synopsis of clinical dermatology. New York: McGraw-Hill, Inc, 1994:242-244.
14. Weston W, Lane A, Morelli J. Color textbook of pediatric dermatology St. Louis, Missouri: Mosby-Year Book, 1996:80-83.

15. Grin C, Rothe MJ. Can you identify these genital lesions? Consultant 2000;40:928-931.

16. Herbst AL, Mishell DR, Stenchever MA, Droegemuller W. Comprehensive gynecology, St. Louis, Missouri: MosbyYear Book, 1992:501.

17. Lim J, Ket Ng S. Oral tetracycline rinse improves symptoms of white sponge nevus. Am Acad of Dermatol 1992;26:1003-1005. 


\section{Author Information}

Nancy H. Busen, PhD, RN, CS, FNP

Associate Professor of Nursing, Coordinator, Graduate PNP Program, School of Nursing, The University of Texas Houston Health Science Center

\section{Marilyn Tompkins, MSN, RN, CS, FNP}

Assistant Professor of Clinical Nursing, School of Nursing, The University of Texas Houston Health Science Center 